\title{
Repeated Leftover Serosurvey of SARS-CoV-2 IgG Antibodies in Greece, May to August 2020
}

\author{
Zacharoula Bogogiannidou ${ }^{1}$, Matthaios Speletas ${ }^{2}{ }^{\circledR}$, Alexandros Vontas ${ }^{1}$, Dimitrios J. Nikoulis ${ }^{1}$, \\ Katerina Dadouli ${ }^{1}$, Maria A. Kyritsi ${ }^{1}$, Varvara A. Mouchtouri ${ }^{1}{ }^{1}$, Paraskevi Mina ${ }^{1}$, Lemonia Anagnostopoulos ${ }^{1}$, \\ Michalis Koureas ${ }^{1}$, Vasileios Karavasilis ${ }^{1}$, Olga Nikou ${ }^{1}$, Ourania Pinaka ${ }^{1}$, Pavlos C. Thomaidis ${ }^{3}$, \\ Kornilia Kadoglou ${ }^{4}$, Konstantinos Bedevis ${ }^{5}$, Natalia Spyrou ${ }^{6}$, Alexandros A. Eleftheriou ${ }^{7}$,

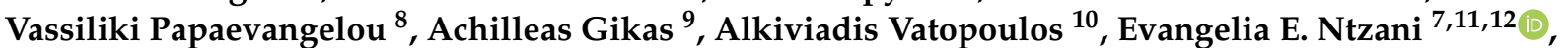 \\ Panagiotis Prezerakos ${ }^{13}$, Sotirios Tsiodras ${ }^{14}$ (D) and Christos Hadjichristodoulou ${ }^{1, *}$
}

Citation: Bogogiannidou, Z.; Speletas, M.; Vontas, A.; Nikoulis, D.J.; Dadouli, K.; Kyritsi, M.A.; Mouchtouri, V.A.; Mina, P.; Anagnostopoulos, L.; Koureas, M.; et al. Repeated Leftover Serosurvey of SARS-CoV-2 IgG Antibodies in Greece, May to August 2020. Vaccines 2021, 9, 504. https:// doi.org/10.3390/vaccines 9050504

Academic Editors: Laurent Verkoczy and Luis Martinez-Sobrido

Received: 17 March 2021

Accepted: 9 May 2021

Published: 13 May 2021

Publisher's Note: MDPI stays neutra with regard to jurisdictional claims in published maps and institutional affiliations.

Copyright: (c) 2021 by the authors. Licensee MDPI, Basel, Switzerland. This article is an open access article distributed under the terms and conditions of the Creative Commons Attribution (CC BY) license (https:// creativecommons.org/licenses/by/ $4.0 /)$.
1 Laboratory of Hygiene and Epidemiology, Faculty of Medicine, University of Thessaly, 41222 Larissa, Greece; xara.16.01@gmail.com (Z.B.); avontas@uth.gr (A.V.); dnikoulis@uth.gr (D.J.N.);

katerina1dad@gmail.com (K.D.); mkiritsi@med.uth.gr (M.A.K.); mouchtourib@med.uth.gr (V.A.M.); pmina@med.uth.gr (P.M.); lanagnost@uth.gr (L.A.); mihaliskoureas@gmail.com (M.K.); karavassilis_v@yahoo.gr (V.K.); onikou@med.uth.gr (O.N.); rpinaka@gmail.com (O.P.)

2 Department of Immunology and Histocompatibility, Faculty of Medicine, University of Thessaly, 41500 Larissa, Greece; maspel@med.uth.gr

3 Microbiological Laboratory “Thomaidis”, 20131 Korinthos, Greece; thomaidispavlos@gmail.com

4 Biochemistry Laboratory, General Hospital of Kalamata, 24150 Kalamata, Greece; kadkoveroia@gmail.com

5 Microbiological Laboratory "Bedevis Konstantinos", 22100 Tripoli, Greece; kkbed@me.com

6 Microbiological Laboratory “Bioiatriki”, 11524 Athens, Greece; nataspyrou@gmail.com

7 Department of Hygiene and Epidemiology, University of Ioannina Faculty of Medicine, 45110 Ioannina, Greece; alexandroseleftheriou4@gmail.com (A.A.E.); entzani@uoi.gr (E.E.N.)

8 Third Department of Paediatrics, School of Medicine, Attikon University Hospital, National and Kapodistrian University of Athens, 12462 Athens, Greece; vpapaev@gmail.com

9 Internal Medicine Department, Infectious Diseases Unit, University Hospital of Heraklion, School of Medicine, University of Crete, 71500 Heraklion, Greece; gikas.achilles@uoc.gr

10 Department of Public Health Policy, School of Public Health, University of West Attica, 11521 Athens, Greece; avatopoulos@uniwa.gr

11 Center for Evidence Synthesis in Health, Department of Health Services, Policy and Practice, School of Public Health, Brown University, Providence, RI 02903, USA

12 Institute of Biosciences, University Research Center of loannina, 45110 Ioannina, Greece

13 Department of Nursing, University of Peloponnese, 22100 Tripoli, Greece; prezerpot@gmail.com

14 Fourth Department of Internal Medicine, School of Medicine, Attikon University Hospital, National and Kapodistrian University of Athens, 12462 Athens, Greece; tsiodras@med.uoa.gr

* Correspondence: xhatzi@med.uth.gr; Tel.: +30-2410565006

Abstract: A serosurvey of IgG antibodies against SARS-CoV-2 was conducted in Greece between May and August 2020. It was designed as a cross-sectional survey and was repeated at monthly intervals. The leftover sampling methodology was used and a geographically stratified sampling plan was applied. Of 20,110 serum samples collected, 89 (0.44\%) were found to be positive for anti-SARS-CoV-2 antibodies, with higher seroprevalence $(0.35 \%)$ observed in May 2020. The highest seroprevalence was primarily observed in the "30-49" year age group. Females presented higher seroprevalence compared to males in May 2020 (females: 0.58\% VS males: $0.10 \%$ ). This difference reversed during the study period and males presented a higher proportion in August 2020 (females: 0.12\% VS males: $0.58 \%$ ). Differences in the rate of seropositivity between urban areas and the rest of the country were also observed during the study period. The four-month infection fatality rate (IFR) was estimated to be $0.47 \%$, while the respective case fatality rate (CFR) was at $1.89 \%$. Our findings confirm low seroprevalence of COVID-19 in Greece during the study period. The young adults are presented as the most affected age group. The loss of the cumulative effect of seropositivity in a proportion of previous SARS-CoV-2 infections was indicated.

Keywords: SARS-CoV-2; COVID-19; antibodies; IgG; seroepidemiology; long-term immune response 


\section{Introduction}

Severe acute respiratory syndrome coronavirus 2 (SARS-CoV-2) is a betacoronavirus and the infectious agent that causes coronavirus disease (COVID-19), a respiratory infection with systemic involvement. The first documented COVID-19 case occurred in Wuhan, China [1] at the end of 2019. Initially, cases were only recorded in Hubei province; however, the COVID-19 outbreak rapidly evolved and was soon characterized as a pandemic by the World Health Organization (WHO) [2].

The first COVID-19 case in Greece was confirmed on 26 February 2020, marking the start of the first pandemic wave in the country, which began in March and lasted until May 2020. During the summer months, some touristic areas within the country recorded a small increase in cases. By 31 August 2020, Greece had reported 8986 laboratory confirmed cases of SARS-CoV-2 infection in the general population and 266 related deaths [3]. The recorded cumulative incidence of COVID-19 in Greece until 31 August was estimated at 87.2 cases per 100,000 population, and the mortality at 2.6 deaths per 100,000 population. The second pandemic wave in Greece began in October 2020.

In an effort to manage the pandemic, countries have applied different strategic interventions with varying levels of success [4]. Extensive diagnostic testing and subsequent interventions are considered essential for controlling and interrupting SARS-CoV-2 transmission. A gradual deterioration of the epidemiological situation in Greece resulted in the implementation of a strict lockdown on 23 March 2020, requiring all residents to limit non-essential movement with minimal exceptions. Beginning on 4 May 2020, public health measures were gradually lifted, with retail/trade businesses the first to reopen, followed by the reopening of schools. As of 1 July 2020, Greece opened its points of entry to tourists.

Measuring host immune response to SARS-CoV-2 infection is an indirect method for detection of COVID-19 beyond the first 2 weeks of illness onset [5]. Considering both the insufficient number of molecular tests conducted and that the majority of individuals infected with SARS-CoV-2 display mild symptoms or remain completely asymptomatic, serological diagnosis is becoming an important tool to understand the extent of COVID19 in the community. Furthermore, serological diagnosis allows for the identification of individuals who are immune and potentially "protected" from becoming infected. The duration of antibody response, particularly in asymptomatic or mild infections is not yet known.

Many scientific articles support the idea of antibodies waning and falling below the threshold of seropositivity approximately two to three months after COVID-19 diagnosis [6,7]. According to the Centers for Disease Control and Prevention (CDC), approximately 2 months after an antibody test, $28 \%$ of seropositive individuals seroreverted to below the threshold of positivity. Thus, point seroprevalence studies could face challenges related to interpretation of results. Conducting repeated serosurveys on a monthly basis using the same sampling methodology could be considered a supplementary surveillance tool.

This seroepidemiological study began in March 2020 and has been repeated at monthly intervals. Results from the first two months have been published elsewhere [8], while here we report on the results from May to August 2020.

The aims of the present seroepidemiological study are to provide an assessment of the extent of COVID-19 spread in the community through estimation of prevalence of SARSCoV-2 IgG antibodies in the Greek population by sex, age group and geographical area; to identify regional, sex and age differences throughout the entire course of the pandemic; and to assess the infection fatality rate (IFR) and compare it to the case fatality rate (CFR). Finally, this serosurvey intends to provide evidence regarding potential under-diagnosis of COVID-19 in Greece. 


\section{Materials and Methods}

\subsection{Study Design and Participants}

The study was designed as a cross-sectional survey and repeated at monthly intervals. We used the leftover sampling methodology in order to collect serum samples (residual sera from the general population) [9]. A geographically stratified sampling plan based on regional units (NUTS level 3) was applied in order to produce a representative sample, taking into consideration age group $(0-29,30-49,50-69$, and $\geq 70$ years $)$ and sex. The required sample size was determined to be 380 serum samples from each of the 13 NUTS level 2 regions and the sample size for each regional unit (NUTS level 3) from the corresponding region was calculated according to population distribution. However, the actual number of collected samples differed from the pre-determined number of samples above.

The leftover serum samples were collected from a nationwide laboratory network, including both private microbiological laboratories as well as microbiological and biochemical laboratories of public hospitals. A total of 36 laboratories participated. The samples were derived from individuals who visited the laboratories for routine screening and reasons unrelated to COVID-19.

The majority of private laboratories, particularly in large urban areas, were closed due to the summer holidays in August 2020, resulting in more challenging sample collection.

\subsection{Laboratory Analysis}

The presence of anti-SARS-CoV-2 IgG antibodies was determined using the ABBOTT SARS-CoV-2 IgG assay, a chemiluminescent microparticle immunoassay (CMIA), with the ARCHITECT i2000SR analyzer (Abbott, Illinois, United States). Anti-spike IgG antibodies are used as a marker of prior SARS-CoV-2 infection. As already stated, the method was validated in our laboratory. We used 305 pre-COVID-19 samples (obtained in 2017) as negative controls and 94 samples from patients with positive SARS-CoV-2 PCR and different symptom durations. The kit displayed $84.0 \%$ sensitivity ( $95 \%$ confidence interval (CI): 76.6-91.5) and 99.7\% specificity (95\% CI: 98.2-100). Given that there were not vaccines during the study period (May to August 2020), all positive samples for IgG anti-SARS-CoV2 were provoked by natural infection.

\subsection{Statistical Analysis}

The statistical analysis applied is identical to the analysis applied for samples between March and April 2020 [8].

\subsection{Weighted Prevalence}

Initially, we determined an unweighted relative frequency of all patient characteristics (age, sex and area of residence): this is the crude seroprevalence (S1). The weighted proportions of positive tests in the countrywide sample were based on the sex and age distribution within each regional unit (NUTS level 3) and the population of each regional unit, according to the most recent census conducted in 2011 (S2) [10]. We also adjusted the weighted proportion (S2) of positive tests to account for the accuracy (sensitivity and specificity) of the laboratory test (S3) [11,12]. Since reported COVID-19 cases were by definition outside the sampling framework, the seroprevalence was corrected, taking into consideration the number of reported cases per month in accordance with the National Public Health Organization (NPHO) (S4). Therefore, we added the cases reported in March, April and May to the estimated S3 seroprevalence in order to calculate the S4 for May, while to calculate the S4 for June we added the reported cases from March to June, and so forth. We calculated the S1, S2, S3 and S4 seroprevalence of IgG antibodies by month and also calculated the CFR and IFR by month. CFR is the ratio of the number of deaths attributed to COVID-19 and reported to the NPHO, divided by the number of cases reported to the NPHO; IFR is the ratio of deaths divided by the number of estimated individuals infected with SARS-CoV-2. The estimation of infected individuals was the product of the seroprevalence and population of regional units where confirmed cases were identified 
according to NPHO [13]. The 95\% CI for weighted data were estimated using normal approximation of binomial distribution and effective sample size, rather than the collected sample size (further explained below). It should be noted that clusters of cases from refugee camps and from a cruise ferry - which were not considered community cases (302 cases in total) - were excluded from analysis for CFR and IFR. The 95\% CI for CFR was calculated using normal approximation of binomial distribution. The 95\% CI for IFR was calculated using the corresponding 95\% CI of the S1, S2, S3 and S4 seroprevalence. Comparison of two proportions was carried out with the ' $\mathrm{N}-1$ ' chi-squared test [13]. In order to calculate how many SARS-CoV-2 infections correspond to one reported case, we estimated the average S3 seroprevalence and corrected it by the estimated $28 \%$ seroconversion according to CDC. For all analyses, a 5\% significance level was set.

\subsection{Effective Sample Size}

Since the number of collected samples from each regional unit was not proportional to the regional unit's population, we calculated an effective sample size based on each regional unit's population proportion, according to 2011 census data. This was done using target weighting. The target sample size for a regional unit $i$ is $t_{i}$, and the actual sample size for the regional unit $i$ is $a_{i}$. The weighting factor for the regional unit $i$ is calculated with the following formula:

$$
\mathrm{f}_{\mathrm{i}}=\frac{\mathrm{t}_{\mathrm{i}}}{\mathrm{a}_{\mathrm{i}}} .
$$

The weighted sample size $\left(\mathrm{w}_{\mathrm{i}}\right)$ for the regional unit $\mathrm{i}$ is calculated as follows:

$$
\mathrm{w}_{\mathrm{i}}=\mathrm{t}_{\mathrm{i}} \times \mathrm{f}_{\mathrm{i}} .
$$

For $\mathrm{k}$ regional units and a countrywide target sample size of $\mathrm{n}_{\mathrm{t}}$, the country-wide effective sample size $\left(n_{e}\right)$ is calculated with the following formula:

$$
\mathrm{n}_{\mathrm{e}}=\frac{\mathrm{n}_{\mathrm{t}}{ }^{2}}{\sum_{\mathrm{i}=1}^{\mathrm{k}} \mathrm{w}_{\mathrm{i}}} .
$$

This can also be written as:

$$
\mathrm{n}_{\mathrm{e}}=\frac{\left(\sum_{\mathrm{i}=1}^{\mathrm{k}} \mathrm{t}_{\mathrm{i}}\right)^{2}}{\sum_{\mathrm{i}=1}^{\mathrm{k}} \frac{\mathrm{t}_{\mathrm{i}}^{2}}{\mathrm{a}_{\mathrm{i}}}} .
$$

\subsection{Ethical Statement}

The samples were anonymized leftover serum samples. Each sample had a unique code and the required data-sex, age, residence and date of blood sampling-were recorded. Health staff from the participating laboratories requested written consent statements from the involved individuals. The research protocol was approved by the ethical committee of the Faculty of Medicine, University of Thessaly, Greece (No. 2116).

\section{Results}

A total of 20,110 samples were collected for the four month period between May and August 2020, of which 11,481 (57\%) were obtained from females. Regarding the age distribution of collected samples, $4375(21.8 \%)$ belonged to the " $0-29$ " age group, $5957(29.6 \%)$ to the "30-49" age group, $5328(26.5 \%)$ to the "50-69" age group and $4547(22.6 \%)$ to individuals " $>70$ " years of age.

Figure 1 displays the geographic distribution of collected leftover samples. A total of 6,054 samples were collected from the Peloponnese region, followed by 3501 from Attica, 1795 from Thessaly, 1748 from Crete, 1510 from Western Greece, 1215 from Eastern Macedonia and Thrace, 1207 from Epirus, 1058 from Western Macedonia, 1011 from Central Macedonia, 677 from Central Greece, 188 from South Aegean and 146 from the Ionian Islands. For each sample, age, sex, residence and date of blood sampling were recorded. 


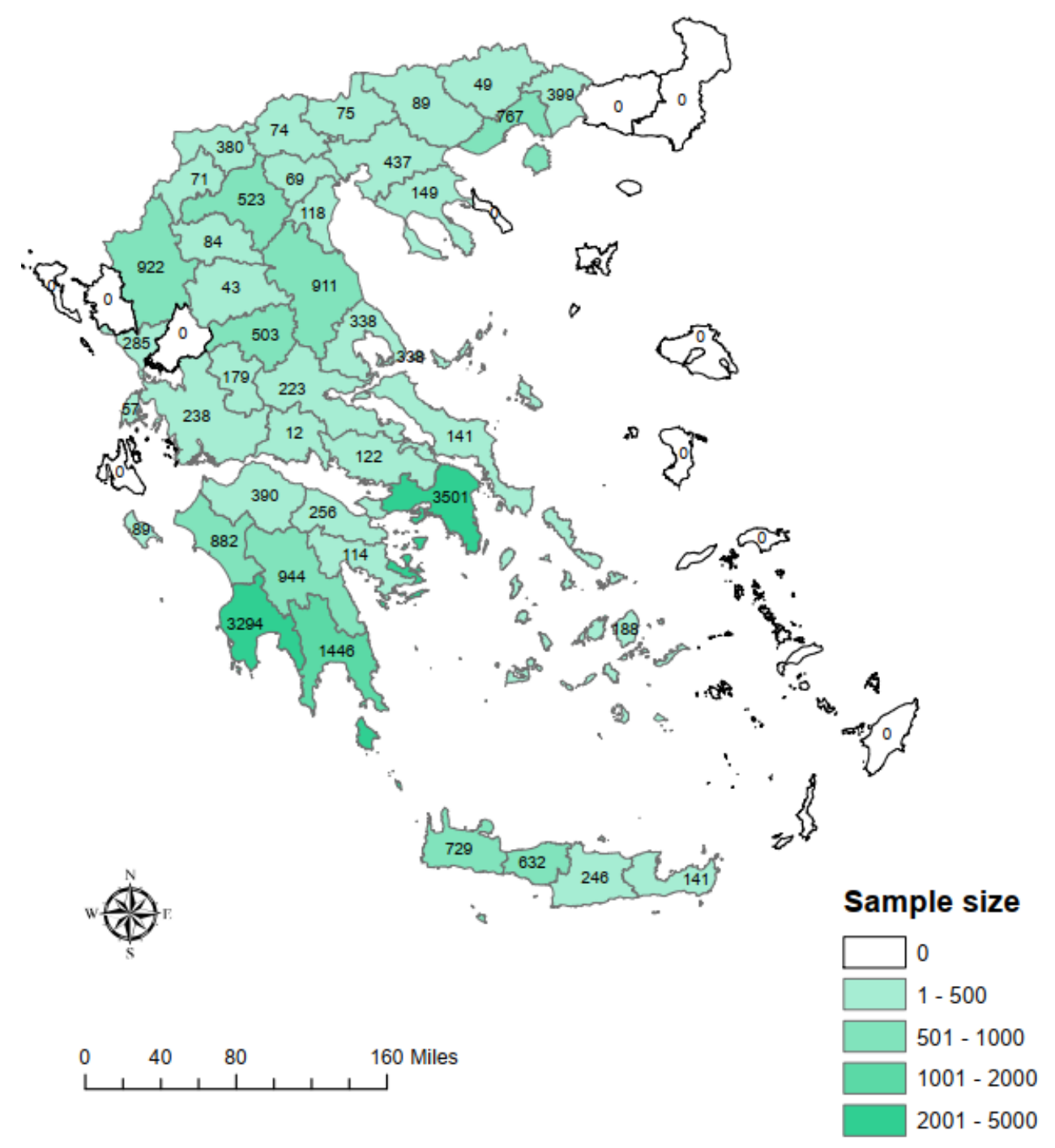

Figure 1. Geographical distribution of leftover samples collected for COVID-19 serosurvey. Some RUs are not covered due to the absence of participating microbiological laboratories.

Of the 20,110 collected serum samples, $89(0.44 \%)$ were found positive for anti-SARSCoV-2 IgG antibodies. According to the monthly distribution of samples, S1 seroprevalence for anti-SARS-CoV-2 IgG antibodies was as follows: $0.44 \%$ in May, $0.37 \%$ in June, $0.49 \%$ in July and $0.52 \%$ in August (Tables 1-4). The adjusted results for age, sex, population (S2) and additionally, for accuracy of the laboratory test (S3) are presented in Tables 1-4. After the addition of NPHO data, S3 seroprevalence was modified and S4 was calculated as $0.35 \%$ in May, $0.19 \%$ in June, $0.25 \%$ in July, and $0.35 \%$ in August (Tables $1-4$ ). 
Table 1. Anti-SARS-CoV-2 IgG antibody seroprevalence, Greece, May $2020(n=5718)$.

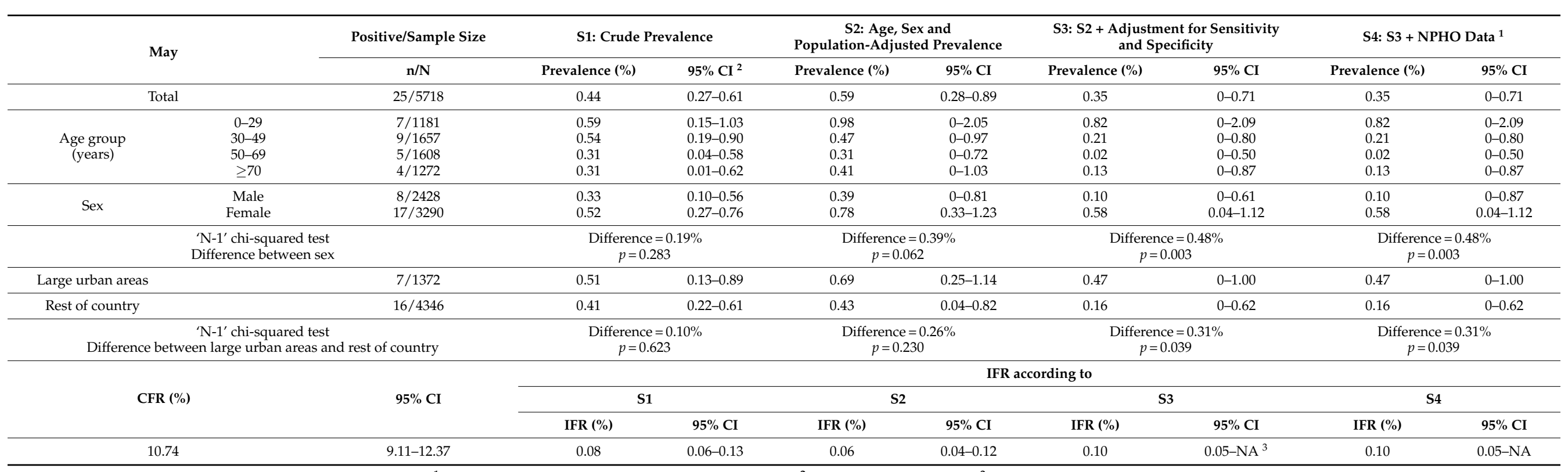

${ }^{1}$ According to NPHO: 326 cases, 35 deaths in May; ${ }^{2}$ CI: confidence interval; ${ }^{3}$ NA: not applicable. 
Table 2. Anti-SARS-CoV-2 IgG antibody seroprevalence, Greece, June $2020(n=6135)$.

\begin{tabular}{|c|c|c|c|c|c|c|c|c|c|c|}
\hline \multirow{2}{*}{\multicolumn{2}{|c|}{ June }} & \multirow{2}{*}{$\begin{array}{c}\text { Positive/Sample Size } \\
\mathbf{n} / \mathbf{N}\end{array}$} & \multicolumn{2}{|c|}{ S1: Crude Prevalence } & \multicolumn{2}{|c|}{$\begin{array}{l}\text { S2: Age, Sex and } \\
\text { Population-Adjusted Prevalence }\end{array}$} & \multicolumn{2}{|c|}{$\begin{array}{c}\text { S3: S2 + Adjustment for Sensitivity and } \\
\text { Specificity }\end{array}$} & \multicolumn{2}{|c|}{ S4: S3 + NPHO Data ${ }^{1}$} \\
\hline & & & Prevalence (\%) & $95 \% \mathrm{CI}$ & Prevalence (\%) & $95 \% \mathrm{CI}$ & Prevalence (\%) & $95 \% \mathrm{CI}$ & Prevalence (\%) & $95 \%$ CI \\
\hline \multicolumn{2}{|c|}{ Total } & $23 / 6135$ & 0.37 & $0.22-0.53$ & 0.46 & $0.17-0.74$ & 0.19 & $0-0.53$ & 0.19 & $0-0.53$ \\
\hline \multirow{4}{*}{$\begin{array}{l}\text { Age group } \\
\text { (years) }\end{array}$} & $0-29$ & $4 / 1366$ & 0.29 & $0.01-0.58$ & 0.22 & $0-0.64$ & 0 & $0-0.40$ & 0 & $0-0.40$ \\
\hline & $30-49$ & $10 / 1885$ & 0.53 & $0.20-0.86$ & 1.16 & $0.32-1.99$ & 1.02 & $0.02-2.02$ & 1.02 & $0.02-2.02$ \\
\hline & $50-69$ & $6 / 1625$ & 0.37 & $0.07-0.66$ & 0.13 & $0-0.45$ & 0 & $0-0.18$ & 0 & $0-0.18$ \\
\hline & $\geq 70$ & $3 / 1259$ & 0.24 & $0-0.51$ & 0.04 & $0-0.28$ & 0 & $0-0.01$ & 0 & $0-0.01$ \\
\hline \multirow[b]{2}{*}{ Sex } & Male & $12 / 2785$ & 0.43 & $0.19-0.67$ & 0.31 & $0-0.67$ & 0.02 & $0-0.44$ & 0.02 & $0-0.44$ \\
\hline & Female & $11 / 3350$ & 0.33 & $0.13-0.52$ & 0.59 & $0.13-1.04$ & 0.34 & $0-0.88$ & 0.34 & $0-0.88$ \\
\hline \multicolumn{2}{|c|}{ Large urban areas } & $7 / 1375$ & 0.51 & $0.13-0.89$ & 0.68 & $0.19-1.18$ & 0.46 & $0-1.05$ & 0.46 & $0-1.05$ \\
\hline \multicolumn{2}{|c|}{ Rest of country } & $16 / 4760$ & 0.34 & $0.17-0.50$ & 0.14 & $0-0.36$ & 0 & $0-0.07$ & 0 & $0-0.07$ \\
\hline \multicolumn{3}{|c|}{$\begin{array}{l}\text { 'N-1' chi-squared test } \\
\text { Difference between large urban areas and rest of country }\end{array}$} & \multicolumn{2}{|c|}{$\begin{array}{l}\text { Difference }=0.17 \% \\
\quad p=0.366\end{array}$} & \multicolumn{2}{|c|}{$\begin{array}{l}\text { Difference }=0.54 \% \\
\quad p<0.001\end{array}$} & \multicolumn{2}{|c|}{$\begin{array}{l}\text { Difference }=0.46 \% \\
\quad p<0.001\end{array}$} & \multicolumn{2}{|c|}{$\begin{array}{l}\text { Difference }=0.46 \% \\
\quad p<0.001\end{array}$} \\
\hline & & & & & & IFR & ording to & & & \\
\hline \multirow{2}{*}{\multicolumn{2}{|c|}{ CFR (\%) }} & $95 \%$ CI & \multicolumn{2}{|c|}{ S1 } & \multicolumn{2}{|c|}{ S2 } & \multicolumn{2}{|c|}{ S3 } & \multicolumn{2}{|c|}{ S4 } \\
\hline & & & IFR (\%) & $95 \% \mathrm{CI}$ & IFR (\%) & $95 \%$ CI & IFR (\%) & $95 \% \mathrm{CI}$ & IFR (\%) & $95 \%$ CI \\
\hline
\end{tabular}

${ }^{1}$ According to NPHO: 377 cases, 17 deaths in June. 
Table 3. Anti-SARS-CoV-2 IgG antibody seroprevalence, Greece, July 2020 ( $n=5959)$.

\begin{tabular}{|c|c|c|c|c|c|c|c|c|c|c|}
\hline \multirow{2}{*}{\multicolumn{2}{|c|}{ July }} & \multirow{2}{*}{$\begin{array}{c}\text { Positive/Sample Size } \\
\mathbf{n} / \mathbf{N}\end{array}$} & \multicolumn{2}{|c|}{ S1: Crude Prevalence } & \multicolumn{2}{|c|}{$\begin{array}{c}\text { S2: Age, Sex and } \\
\text { Population-Adjusted Prevalence }\end{array}$} & \multicolumn{2}{|c|}{$\begin{array}{l}\text { S3: S2 + Adjustment for Sensitivity and } \\
\text { Specificity }\end{array}$} & \multicolumn{2}{|c|}{ S4: S3 + NPHO Data ${ }^{1}$} \\
\hline & & & Prevalence (\%) & $95 \% \mathrm{CI}$ & Prevalence (\%) & $95 \% \mathrm{CI}$ & Prevalence (\%) & $95 \% \mathrm{CI}$ & Prevalence (\%) & $95 \% \mathrm{CI}$ \\
\hline \multicolumn{2}{|c|}{ Total } & $29 / 5959$ & 0.49 & $0.31-0.66$ & 0.50 & $0.24-0.76$ & 0.24 & $0-0.55$ & 0.25 & $0.01-056$ \\
\hline \multirow{4}{*}{$\begin{array}{l}\text { Age group } \\
\text { (years) }\end{array}$} & $0-29$ & $6 / 1221$ & 0.49 & $0.10-0.88$ & 0.32 & $0-0.74$ & 0.02 & $0-0.52$ & 0.02 & $0-0.52$ \\
\hline & $30-49$ & $8 / 1645$ & 0.49 & $0.15-0.82$ & 0.69 & $0.13-1.25$ & 0.46 & $0-1.13$ & 0.47 & $0.01-1.14$ \\
\hline & $50-69$ & $9 / 1586$ & 0.57 & $0.20-0.94$ & 0.56 & $0.03-1.09$ & 0.31 & $0-0.95$ & 0.32 & $0.01-0.96$ \\
\hline & $\geq 70$ & $6 / 1507$ & 0.40 & $0.08-0.72$ & 0.44 & $0-1.01$ & 0.16 & $0-0.84$ & 0.17 & $0.01-0.85$ \\
\hline \multirow[b]{2}{*}{ Sex } & Male & $17 / 2442$ & 0.70 & $0.37-1.03$ & 0.52 & $0.11-0.93$ & 0.26 & $0-0.75$ & 0.27 & $0.01-0.76$ \\
\hline & Female & $12 / 3517$ & 0.34 & $0.15-0.53$ & 0.48 & $0.15-0.82$ & 0.22 & $0-0.62$ & 0.23 & $0.01-0.63$ \\
\hline \multicolumn{2}{|c|}{ Large urban areas } & $10 / 1593$ & 0.63 & $0.24-1.02$ & 0.53 & $0.15-0.91$ & 0.28 & $0-0.73$ & 0.29 & $0.01-0.74$ \\
\hline \multicolumn{2}{|c|}{ Rest of country } & $19 / 4366$ & 0.44 & $0.24-0.63$ & 0.47 & $0.13-0.81$ & 0.20 & $0-0.61$ & 0.21 & $0.01-0.62$ \\
\hline \multicolumn{3}{|c|}{$\begin{array}{l}\text { 'N-1' chi-squared test } \\
\text { Difference between large urban areas and rest of country }\end{array}$} & \multicolumn{2}{|c|}{$\begin{array}{l}\text { Difference }=0.19 \% \\
\quad p=0.353\end{array}$} & \multicolumn{2}{|c|}{$\begin{array}{l}\text { Difference }=0.06 \% \\
\quad p=0.768\end{array}$} & \multicolumn{2}{|c|}{$\begin{array}{l}\text { Difference }=0.08 \% \\
\qquad p=0.561\end{array}$} & \multicolumn{2}{|c|}{$\begin{array}{l}\text { Difference }=0.08 \% \\
\quad p=0.570\end{array}$} \\
\hline & & & & & & IFR & ording to & & & \\
\hline \multirow{2}{*}{\multicolumn{2}{|c|}{ CFR (\%) }} & $95 \% \mathrm{CI}$ & \multicolumn{2}{|c|}{ S1 } & \multicolumn{2}{|c|}{ S2 } & \multicolumn{2}{|c|}{ S3 } & \multicolumn{2}{|c|}{ S4 } \\
\hline & & & IFR (\%) & $95 \% \mathrm{CI}$ & IFR (\%) & $95 \% \mathrm{CI}$ & IFR (\%) & $95 \% \mathrm{CI}$ & IFR (\%) & $95 \% \mathrm{CI}$ \\
\hline
\end{tabular}

${ }^{1}$ According to NPHO: 700 cases, 14 deaths in July. 
Table 4. Anti-SARS-CoV-2 IgG antibody seroprevalence, Greece, August $2020(n=2298)$.

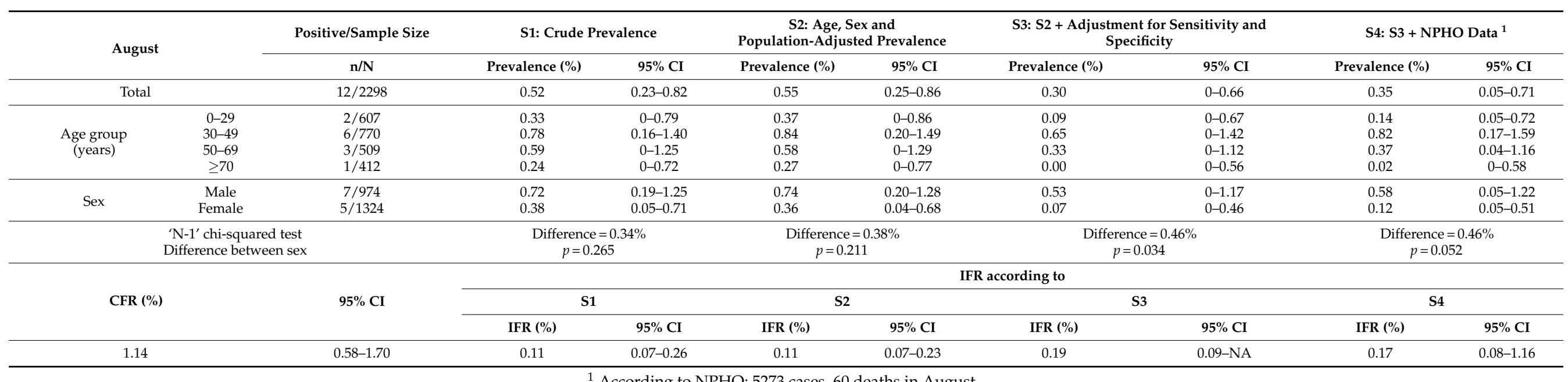

${ }^{1}$ According to NPHO: 5273 cases, 60 deaths in August. 
Throughout the study period, the two younger age groups presented the highest seroprevalence. Specifically, in May 2020 the highest seroprevalence was observed in the " $0-29$ " year age group with S4 $=0.82 \%$, while in June, July and August 2020 the highest seroprevalence was estimated in the " $30-49$ " year age group with $\mathrm{S} 4=1.02 \%, 0.47 \%$, and $0.82 \%$, respectively (Tables $1-4$, Figure 2 ).

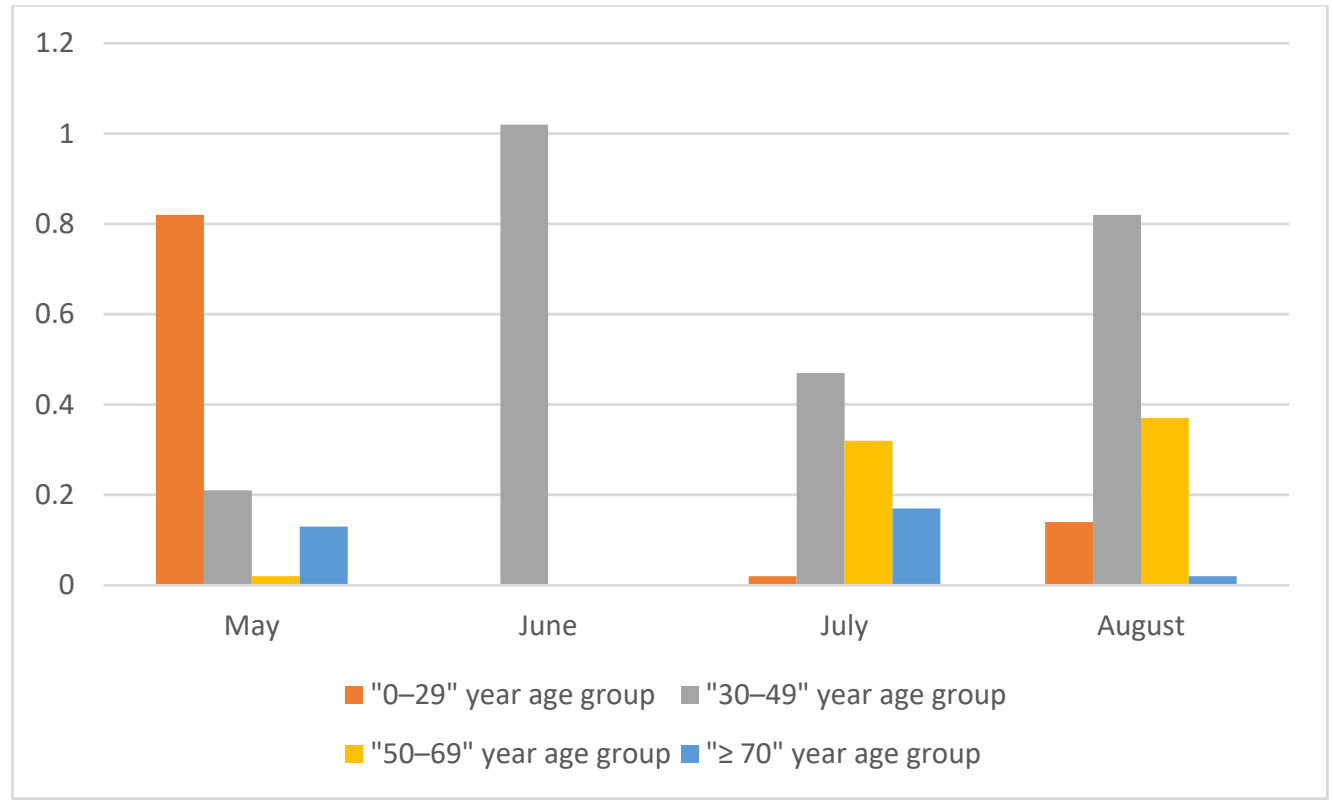

Figure 2. Seroprevalence by age group between May to August 2020.

During the first two months of the study period, higher seroprevalence was estimated in large urban areas (Attica region and the regional unit of Thessaloniki) as compared to the rest of the country. In large urban areas, the $\mathrm{S} 4$ was calculated as $0.47 \%$ and $0.46 \%$ in May and June respectively, while in the rest of the country the $\mathrm{S} 4$ was calculated as $0.16 \%$ and $0 \%$ for the corresponding months (May: difference $=0.31 \%, p=0.039$; June: difference $=0.46 \%$, $p<0.0001$ ) (Tables 1 and 2). However, in July 2020 the S4 was calculated as $0.29 \%$ in large urban areas and $0.21 \%$ for the rest of the country, with no statistically significant difference estimated $(p=0.570)$. Due to an insufficient number of samples collected from large urban areas in month of August, due to closures of many microbiological laboratories during summer holidays, seroprevalence was not calculated separately based on areas by population density.

The S4 was higher among females as compared to males in May and June (May: females: S4 $=0.58 \%$ VS males: S4 $=0.10 \%$, June: females: S4 $=0.34 \%$, VS males: $\mathrm{S} 4=0.02 \%)$ (Tables 1 and 2); however, this difference was reversed during July and August (July: females: S4 $=0.23 \%$ VS males: S4 $=0.27 \%$, August: females: S4 $=0.12 \%$ VS males: S4 $=0.58 \%$ ) (Figure 3). The difference between sexes observed in August $(0.46 \%)$ is of borderline statistical significance $(p=0.052)$. Additionally, seroprevalence in each age group was calculated separately for each sex (Table S1). 


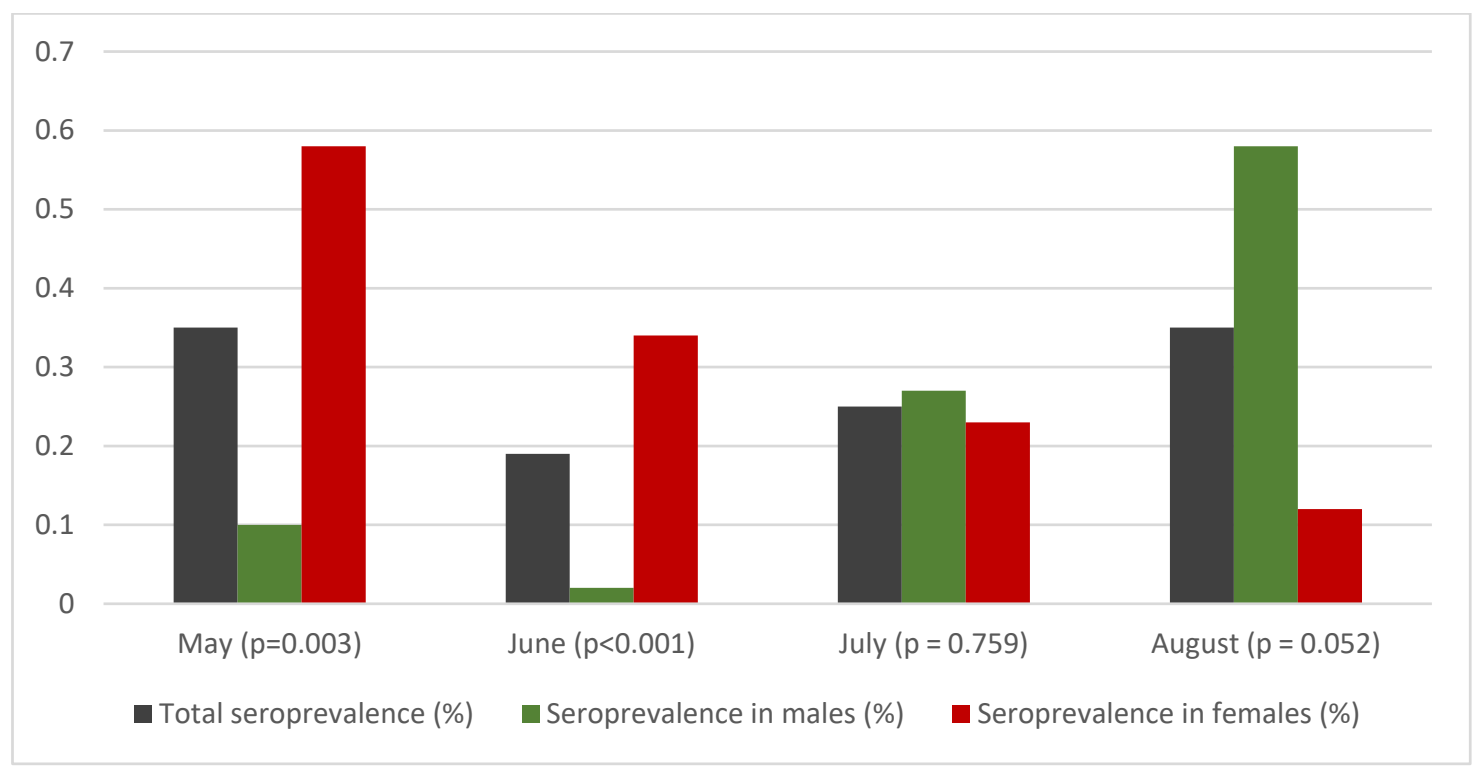

Figure 3. Total seroprevalence, and seroprevalence per sex from May to August 2020 ( $p$ indicates the difference between sexes).

Summarizing the above results, for the months of May and June, statistically significant difference was observed between the two sexes (May $p=0.003$ and June $p<0.001$ ) and between large urban areas and rest of the country (May $=0.039$ and June $p<0.001$ ) with females and large urban areas presented higher seroprevalence. These differences were not observed in July. In August, a borderline no statistically significant difference was calculated $(p=0.052)$ between males and females, with $S 4=0.58 \%$ in males and $0.12 \%$ in females.

Tables 1-4 present the monthly CFR and IFR. A gradual decline in CFR was observed, while IFR remained below $0.2 \%$ each month during the study period, presenting a slight difference from month to month. Between May and August 2020, 126 deaths were reported according to data from the NPHO. Consequently, the CFR for this 4-month period was calculated as $1.89 \%$ and the corresponding IFR as $0.47 \%$ (Table 5).

Table 5. Anti-SARS-CoV-2 IgG antibody seroprevalence, IFR, CFR, Greece, May-August 2020.

\begin{tabular}{|c|c|c|c|c|c|c|c|c|c|}
\hline \multirow{2}{*}{ May-August } & \multirow{2}{*}{$\begin{array}{c}\text { Positive/ } \\
\text { Sample Size } \\
\text { n/N }\end{array}$} & \multicolumn{2}{|c|}{ S1: Crude Prevalence } & \multicolumn{2}{|c|}{$\begin{array}{c}\text { S2: Age, Sex and } \\
\text { Population-Adjusted } \\
\text { Prevalence }\end{array}$} & \multicolumn{2}{|c|}{$\begin{array}{l}\text { S3: S2 + ;Adjustment for } \\
\text { Sensitivity and Specificity }\end{array}$} & \multicolumn{2}{|c|}{ S4: S3 + NPHO Data } \\
\hline & & $\begin{array}{l}\text { Prevalence } \\
\text { (\%) }\end{array}$ & $95 \% \mathrm{CI}$ & $\begin{array}{l}\text { Prevalence } \\
(\%)\end{array}$ & $95 \% \mathrm{CI}$ & $\begin{array}{l}\text { Prevalence } \\
(\%)\end{array}$ & $95 \%$ CI & $\begin{array}{l}\text { Prevalence } \\
\text { (\%) }\end{array}$ & $95 \% \mathrm{CI}$ \\
\hline Total & $89 / 20,110$ & 0.44 & $0.35-0.53$ & 0.46 & $0.37-0.55$ & 0.19 & $0.08-0.30$ & 0.26 & $0.15-0.37$ \\
\hline \multirow{3}{*}{ CFR (\%) } & \multirow{3}{*}{$95 \%$ CI } & \multicolumn{8}{|c|}{ IFR according to } \\
\hline & & \multicolumn{2}{|c|}{ S1 } & \multicolumn{2}{|c|}{$\mathrm{S} 2$} & \multicolumn{2}{|c|}{ S3 } & \multicolumn{2}{|c|}{$\mathrm{S} 4$} \\
\hline & & IFR (\%) & $95 \%$ CI & IFR (\%) & $95 \% \mathrm{CI}$ & IFR (\%) & $95 \%$ CI & IFR (\%) & $95 \% \mathrm{CI}$ \\
\hline 1.89 & $1.56-2.21$ & 0.28 & $0.23-0.35$ & 0.27 & $0.22-0.33$ & 0.63 & $0.40-1.51$ & 0.47 & $0.33-0.84$ \\
\hline
\end{tabular}

A total of 5094 new cases were reported between May and August 2020 in the general population, excluding the Attica region. By using the average S3 seroprevalence for this time period and increasing it by $28 \%$ (the percentage of seropositives that seroconvert), according to the CDC it was estimated that each case reported by the NPHO corresponded to 4.9 SARS-CoV-2 infections in the general Greek population (95\% CI: 2.1-7.8). 


\section{Discussion}

Our results demonstrate low seroprevalence of anti-SARS-CoV-2 IgG antibodies in Greece during the first wave of the pandemic. This finding supports hypotheses regarding the effectiveness of the timely implementation of public health measures. In March and April 2020, the serosurvey results were calculated as S4 $=0.02 \%$ and $0.25 \%$, respectively [8]. In Figure 4, we summarize our previous and current serosurvey results, in addition to presenting the monthly cases according to NPHO data. A peak in seroprevalence was observed in May 2020; this peak can be explained as representing the proportion of individuals infected with SARS-CoV-2 during the first pandemic wave between March to May 2020. Consequently, an increase in seroprevalence would be expected in May, as compared to March and April 2020. Figure 4 becomes more remarkable, taking into consideration the different public health measures implemented during this period. From 23 March 2020 a national lockdown had been imposed, which was gradually lifted beginning 4 May 2020, with a requirement for masks to be worn in areas of intense crowding (such as on means of public transport, at supermarkets, hospitals etc.). Furthermore, on 18 May mobility between regional units within the country was permitted. Between mid-May to June, schools gradually reopened with senior classes the first to commence. In late May, eating establishments were reopened and starting from 1 July 2020, the entry of tourists into Greece from all countries was permitted.

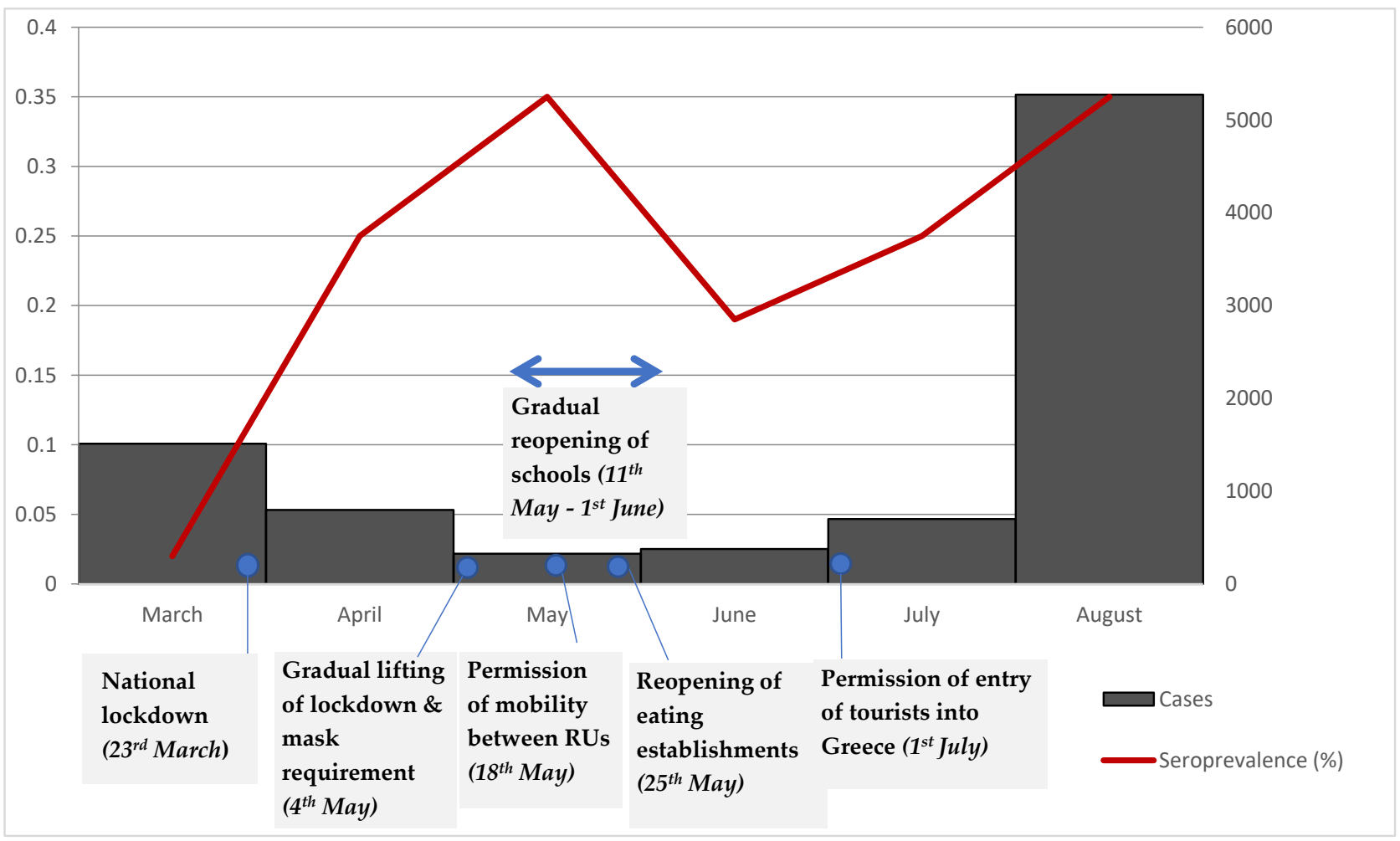

Figure 4. The seroprevalence, monthly cases and main public health measures in Greece during the period from March to August 2020.

A cumulative effect of seropositivity would be conventional, increasing even with lower positivity rates over the following months. However, the seroprevalence in June is lower than the seroprevalence in May. This finding has been documented by corresponding seroepidemological studies reflecting antibody waning [6,14].

Our positive results reflect mainly mild or asymptomatic cases, since confirmed COVID-19 cases that occurred during the same month were excluded from the sampling framework. It is widely supported that both anti-SARS-CoV-2 antibody titers and their duration of detection are correlated with the severity of clinical presentation [15]. Mild 
COVID-19 cases develop antibodies in a lower titer and for a shorter time period. The halflife of anti-SARS-CoV-2 antibodies has been calculated as 106 days [7] and, subsequently, several of them end in "serosilent" infections.

As mentioned above, the CDC suggests that, approximately 2 months after the first detection of antibodies, nearly one of four seropositive individuals seroconverted below the threshold of positivity [6]. This documented waning of antibodies following infection decreases the sensitivity of antibody detection throughout the months. Considering this, the observed decrease of seroprevalence in June 2020 can be justified by both the reduced spread of SARS-CoV-2 in the community and the simultaneous seroconversion of seropositive individuals infected in March and April 2020.

This decrease in seroprevalence raises concerns related to long-term immune response. A few reports exist that describe cases of reinfection; however, this occurrence is extremely rare [16]. In the following months between July and August 2020, a small increase in seroprevalence was observed. This finding may be explained by the unrestricted movement of citizens during this period and increased travel due to summer holidays.

During the summer months, it is well-recognized and customary for the general Greek population to travel from large urban areas to areas throughout the rest of the country. This "pattern of mobility" may offer an explanation for our second remarkable finding, regarding the diminished differences in seroprevalence between these two areas. In May and June 2020, the calculated seroprevalence was higher in large urban areas compared to the rest of the country, while almost the same seroprevalence was estimated in July 2020 for both areas. This finding reflects the spread of SARS-CoV-2 in non-urban areas due to population movement for summer holidays, after the remaining movement restrictions were lifted for the general population on 25 May 2020 Individuals from large urban areas where higher SARS-CoV-2 spread had been observed travelled to their holiday destinations throughout the rest of country, where the susceptible local populations were at risk of becoming infected. This "population movement" resulted in a lessening of seroprevalence differences between the two areas.

Unfortunately, a sufficient number of samples from large urban areas for August 2020 were unavailable. However, the existence of a difference in seroprevalence between large urban areas and the rest of the country will be further examined in the following months, as the collection of samples continued from September 2020 onward according to the established methodology.

In contrast to our previous results presenting a simultaneous increase in seropositivity with increasing age, seroprevalence between May and August 2020 was primarily higher in the "30-49" year age group [8]. This finding could be attributed to the fact that Greece's workforce is largely comprised of individuals in this age group and, furthermore, increased mobility is associated with this subset of the population due to their more active participation in recreational activities. Considering the above factors and that younger age groups that become infected with COVID-19 typically experience mild disease, may offer an explanation for the high seroprevalence observed in this age group [17].

Continuous communications campaigns provided by public health authorities regarding the protection of elderly populations likely influenced the behavior of elderly individuals after restrictive public health measures were lifted. This protective behavior could be considered as a reason for the low seroprevalence observed in the " $>70$ " year age group. The results of the next months will enrich the discussion of this issue.

Throughout the months, an increase in seroprevalence in males compared to females can be observed. This finding is consistent with data from the NPHO (55.6\% of cases refer to males). Interpretation of this result must also consider that males comprise a large majority of the workforce in Greece and tend to participate more actively in recreational activities.

Both findings related to age, group and sex are consistent with the results of a published systematic review and meta-analysis, which included preprints or peer-reviewed articles up to 14 August 2020. Higher seroprevalence is calculated in males compared to 
females. Moreover, in this review the "20-49" year age group shows the highest prevalence, followed by the "50-65" year age group [18].

A total of 2310 COVID-19 cases were reported by the NPHO up to 30 April 2020, and it was calculated that one reported COVID-19 case corresponded to ten SARS-CoV-2 infections in the Greek general population [8]. For the following months, the corresponding factor decreased to 4.9. This reduction is justified by an increase in the number of tests conducted and therefore, increased detection of COVID-19 cases. A similar ratio of approximately 4 was identified when comparing the estimated CFR with the IFR for this four-month period. It should be noted that the delay of death could not be accounted for in the current estimation.

The leftover sampling methodology could be considered a limitation of the study, as non-random convenient sampling may affect the representativeness of samples collected. A limitation of the leftover sampling methodology is the difficulty related to collection of clinical information. Using this methodology, it was therefore not possible to correlate clinical status with positive samples. Sample collection was also challenging due to summer closures of many microbiological laboratories, primarily in August 2020. Not all areas were covered by the sampling network.

This methodology has several advantages including ease of sample collection. Furthermore, this methodology allows for repeated monthly sample collection, enabling follow up of the course of the pandemic and immunity levels of the general population on a rolling basis.

\section{Conclusions}

Our study presents low seroprevalence in Greece during the period from May to August 2020, a finding which renders the Greek population extremely vulnerable to SARSCoV-2 infection. The " $30-49$ " year age group appears to have been most affected during this period, since this subset of the population is characterized by the highest mobility levels and most actively participates in recreational activities. The differences in seroprevelance between large urban areas and other areas throughout the country lessened, and towards the end of the study period, a higher seroprevalence in males was observed. As was expected, a lower IFR was calculated compared to CFR. The waning of anti-SARS-CoV-2 IgG antibodies is supported by our serosurvey results.

Supplementary Materials: The following are available online at https:/ / www.mdpi.com/article/ 10.3390/vaccines9050504/s1, Table S1: S1 and S1 adjusted for Se \& Sp in each age group for each sex separately.

Author Contributions: Conceptualization, C.H., M.S., S.T., P.P. and Z.B.; Methodology, C.H. and M.S.; Formal Analysis, C.H. and K.D.; Resources, E.E.N., A.V. (Alkiviadis Vatopoulos), D.J.N., A.A.E., V.K., P.M., O.N., O.P., P.C.T., K.K., K.B. and N.S.; Data Curation, Z.B.; Writing-Original Draft Preparation, C.H. and Z.B.; Writing-Review \& Editing, L.A., V.A.M., M.K., E.E.N. and C.H. Visualization, K.D.; Supervision, C.H., M.S., S.T., P.P., A.G., V.P., V.A.M. and M.A.K.; Project Administration, A.V. (Alexandros Vontas) and Z.B. All authors have read and agreed to the published version of the manuscript.

Funding: The study was funded by the Greek Ministry of Health.

Institutional Review Board Statement: The study was conducted according to the guidelines of the Declaration of Helsinki and was approved by the by the ethical committee of the Faculty of Medicine, University of Thessaly, Greece (No. 2116, 22.04.2020).

Informed Consent Statement: Informed consent was obtained from all subjects involved in the study.

Acknowledgments: The authors highly appreciate the continuous support for this project offered by the laboratories that participated in the study. Moreover, the authors would like to thank the National Public Health Organization for providing surveillance data on COVID-19. 
Conflicts of Interest: The authors declare no conflict of interest. The funders had no role in the design of the study; in the collection, analyses, or interpretation of data; in the writing of the manuscript, or in the decision to publish the results.

\section{References}

1. Chan, J.F.W.; Yuan, S.; Kok, K.H.; To, K.K.W.; Chu, H.; Yang, J.; Xing, F.; Liu, J.; Yip, C.C.Y.; Poon, R.W.S.; et al. A familial cluster of pneumonia associated with the 2019 novel coronavirus indicating person-to-person transmission: A study of a family cluster. Lancet 2020, 395, 514-523. Available online: https:/ / www.sciencedirect.com/science/article/pii/S0140673620301549 (accessed on 4 March 2021). [CrossRef]

2. WHO Director-General's Opening Remarks at the Media Briefing on COVID-19-11 March 2020. Available online: https:/ / www.who.int/director-general/speeches/detail/who-director-general-s-opening-remarks-at-the-media-briefing-oncovid-19---11-march-2020 (accessed on 4 March 2021).

3. Greece: Coronavirus Pandemic Country Profile-Our World in Data. Available online: https://ourworldindata.org/coronavirus/ country/greece\#what-is-the-cumulative-number-of-confirmed-deaths (accessed on 16 April 2021).

4. Policy Responses to the Coronavirus Pandemic-Statistics and Research-Our World in Data. Available online: https: / / ourworldindata.org/policy-responses-covid (accessed on 4 March 2021).

5. Sethuraman, N.; Jeremiah, S.S.; Ryo, A. Interpreting Diagnostic Tests for SARS-CoV-2. JAMA 2020, 323, 2249-2251. [CrossRef] [PubMed]

6. Self, W.H.; Tenforde, M.W.; Stubblefield, W.B.; Feldstein, L.R.; Steingrub, J.S.; Shapiro, N.I.; Ginde, A.A.; Prekker, M.E.; Brown, S.M.; Peltan, I.D.; et al. Decline in SARS-CoV-2 Antibodies After Mild Infection Among Frontline Health Care Personnel in a Multistate Hospital Network-12 States, April-August 2020. MMWR Morb. Mortal Wkly. Rep. 2020, 69, 1762-1766. [CrossRef] [PubMed]

7. Buss, L.F.; Prete, C.A.; Abrahim, C.M.; Mendrone, A.; Salomon, T.; de Almeida-Neto, C.; França, R.F.; Belotti, M.C.; Carvalho, M.P.; Costa, A.G.; et al. Three-quarters attack rate of SARS-CoV-2 in the Brazilian Amazon during a largely unmitigated epidemic. Science 2021, 371, 288-292. Available online: http:/ / science.sciencemag.org/content/371/6526/288.abstract (accessed on 4 March 2021). [CrossRef] [PubMed]

8. $\quad$ Bogogiannidou, Z.; Vontas, A.; Dadouli, K.; Kyritsi, M.A.; Soteriades, S.; Nikoulis, D.J.; Mouchtouri, V.A.; Koureas, M.; Kazakos, E.I.; Spanos, E.G.; et al. Repeated leftover serosurvey of SARS-CoV-2 IgG antibodies, Greece, March and April 2020. Eurosurveillance 2020, 25, 1-6. [CrossRef] [PubMed]

9. Nardone, A.; Miller, E. Serological surveillance of rubella in Europe: European Sero-Epidemiology Network (ESEN2). Eurosurveilllance 2004, 9, 5-7. [CrossRef] [PubMed]

10. Naing, N.N. Easy way to learn standardization: Direct and indirect methods. Malays. J. Med. Sci. 2000, 7, 10-15. [PubMed]

11. Bendavid, E.; Mulaney, B.; Sood, N.; Shah, S.; Ling, E.; Bromley-Dulfano, R.; Lai, C.; Weissberg, Z.; Saavedra-Walker, R.; Tedrow, J.; et al. COVID-19 Antibody Seroprevalence in Santa Clara County, California. MedRxiv 2020. Available online: http:/ / medrxiv.org/content/early/2020/04/30/2020.04.14.20062463.abstract (accessed on 4 March 2021). [CrossRef]

12. Diggle, P.J. Estimating Prevalence Using an Imperfect Test. Epidemiol. Res. Int. 2011, 2011, 608719. [CrossRef]

13. Campbell, I. Chi-squared and Fisher-Irwin tests of two-by-two tables with small sample recommendations. Stat. Med. 2007, 26, 3661-3675. Available online: https:/ / pubmed.ncbi.nlm.nih.gov/17315184/ (accessed on 4 March 2021). [CrossRef]

14. Moshe, M.; Brown, J.C.; Flower, B.; Daunt, A.; Ward, H.; Elliott, P. Declining prevalence of antibody positivity to SARS-CoV-2: A community study of 365,000 adults. MedRxiv 2020. [CrossRef]

15. Hansen, C.B.; Jarlhelt, I.; Pérez-Alós, L.; Landsy, L.H.; Loftager, M.; Rosbjerg, A.; Helgstrand, C.; Bjelke, J.R.; Egebjerg, T.; Jardine, J.G.; et al. SARS-CoV-2 Antibody Responses Are Correlated to Disease Severity in COVID-19 Convalescent Individuals. J. Immunol. 2021, 206, 109-117. Available online: http://www.jimmunol.org/content/206/1/109.abstract (accessed on 4 March 2021). [CrossRef] [PubMed]

16. To, K.K.W.; Hung, I.F.N.; Ip, J.D.; Chu, A.W.H.; Chan, W.M.; Tam, A.R.; Fong, C.H.Y.; Yuan, S.; Tsoi, H.W.; Ng, A.C.K.; et al. COVID-19 re-infection by a phylogenetically distinct SARS-coronavirus-2 strain confirmed by whole genome sequencing. Clin. Infect. Dis. 2020, ciaa1275. Available online: https:/ / pubmed.ncbi.nlm.nih.gov/32840608 (accessed on 4 March 2021).

17. Bajema, K.L.; Wiegand, R.E.; Cuffe, K.; Patel, S.V.; Iachan, R.; Lim, T.; Lee, A.; Moyse, D.; Havers, F.P.; Harding, L.; et al. Estimated SARS-CoV-2 Seroprevalence in the US as of September 2020. JAMA Intern. Med. 2020, 181, 450-460. [CrossRef]

18. Rostami, A.; Sepidarkish, M.; Leeflang, M.; Riahi, S.M.; Shiadeh, M.N.; Esfandyari, S.; Mokdad, A.H.; Hotez, P.J.; Gasser, R.B. SARS-CoV-2 seroprevalence worldwide: A systematic review and meta-analysis. Clin. Microbiol. Infect. 2020, 27, 331-340. [CrossRef] [PubMed] 